\title{
XXXIV. Theoretical explanations of the rectilinear transmission and spontaneous diffusion of sound and light
}

\author{
Professor Challis M.A. F.R.S. F.R.A.S.
}

To cite this article: Professor Challis M.A. F.R.S. F.R.A.S. (1881) XXXIV. Theoretical explanations of the rectilinear transmission and spontaneous diffusion of sound and light, Philosophical Magazine Series 5, 11:68, 249-254, DOI: $10.1080 / 14786448108627009$

To link to this article: http://dx.doi.org/10.1080/14786448108627009

册 Published online: 08 Jun 2010.

Submit your article to this journal $\pi$

Џ Article views: 2

Q View related articles $\longleftarrow$ 
Clausius, in the paper previously referred to, explains the various phenomena produced by currents by means of this law of force, and the hypothesis that a current consists of streams of opposite electricities moving in opposite directions. Now, since the expressions we have obtained for the force between the particle do not depend on the specific inductive capacity of the medium, but only on its magnetic permeability, if we make this assumption about the nature of a current, it follows from Maxwell's theory that the electrodynamic phenomena produced by a current of given strength do not depend on the specific inductive capacity of the surrounding medium, though they do depend on its magnetic permeability.

Faraday, in his 'Experimental Researches' ( $\$ 1709$ and onwards), describes some experiments which he made to determine whether altering the surrounding medium produced any change in the electromagnetic action of a current. The result of the experiments was that he was unable to detect any such change; but in his experiments, though the specific inductive capacities of the various media tried were very different, their magnetic permeabilities were all of them very nearly unity.

XXXIV. Theoretical Explanations of the Rectilinear Transmission and Spontaneous Diffusion of Sound and Light. $B y$ Professor Chaldis, M.A., F.R.S., F.R.A.S.*

WHEN any disturbance is produced at a given position $A$ in an unlimited mass of elastic fluid of perfect fluidity, defined by the relation $p=a^{2} \rho$ between its pressure $p$ and density $\rho, a^{2}$ being constant, it is found by experience that there will be a resulting state of the fluid at a point $P$, whose position is taken ad libitum, and at all intermediate points between $A$ and $P$. In other words, there will be a rectilinear transmission of effect from $A$ to all surrounding points, without respect to the particular mode of disturbing the fluid. It may be that a difference of effect in different directions may depend on the mode of disturbance; at the same time it is found that a resulting disturbance is produced at all points, whatever be the mode of disturbance. In treatises on hydrodynamics this remarkable fact is left out of consideration. I know of none in which this problem has been solved, or eren proposed. But it is an admitted principle that when the fundamentals of any branch of applied science, after being established by observation and experiment, have been ex-

* Communicated by the Author. 
pressed by general equations, all other related facts of observation admit of being accounted for by mathematical reasoning derived from the equations. The science of Analytical Hydrodynamics must be pronounced to be in a very imperfect state, unless it be capable of giving a reason for such a patent and general fact as that just mentioned. The argument I am about to adduce is intended to give the required explanation. It will be proper to premise here that this argument has nothing to do with the necessity I have so often insisted upon, of a third general equation for completing the analytical principles of hydrodynamics. I still maintain that the principles on which that equation rests, and the process of its deduction, are so simple and direct that they do not admit of being controverted; but they do not come under consideration in the present enquiry.

Supposing the reasoning to apply to a portion of the fluid contained in a straight rectangular tubs of small uniform transverse section extending from $A$ to $P$, it is evident that if arbitrary motion be communicated to the fluid by a movable diaphragm at $A$ exactly fitting the tabe, rectilinear motion will take place in the direction of the axis of the tube. This problem has been discussed by Poisson in the Journal de l'Ecole Polytechnique, tom. vii., for the case in which $p=a^{2} \rho$; and from the mathematical investigation he deduces a particular integral from which he infers (p. 369) that the original disturbance will be transmitted uniformly with a velocity equal to $a$. The same problem has been solved by Mr. Earnshaw, in the Philosophical Transactions for $1860, \mathrm{p} .133$, by a process differing only in form from that of Poisson, and conducting to the same results. From this integral I obtained in a particular case the absurd conclusion that the same particle of fluid might be at rest and have a maximum motion at the same instant of time (see Philosophical Magazine for June 1848, p. 496, and my 'Principles of Mathematics and Physies,' p. 195). The reasoning by which this result was reached is so certain that it does not admit of being questioned, and, in fact, has not been directly questioned; but Mr. Earnshaw has thought good to say, that the wave "will force its way in violation of our equations." This is so strange and inadmissible an assertion that $I$ have a right to regard it as a virtual acknowledgment of the reductio ad absurdum above mentioned, the reality of which I shall accordingly take for granted.

But a reductio ad absurdum is not a result which can be slurred over. It is absolutely necessary for the satisfactory establishment of the principles of any science in which it 
occurs, that the absurdity should be accounted for and got rid of. I have adverted above to two modes in which a disturbance at $A$ may be rectilinearly transmitted from $A$, so as to have effect at any point $P$. In the first it was stated that, as matter of experience, the effect takes place independently of any particular conditions under which the fluid was disturbed at A; in the other the fluid is assumed to be disturbed in a particular manner, and under certain conditions. The former of these modes may be called, for distinction, free or spontaneous motion, and the other constrained or arbitrary motion. Now, in the received analytical theory of hydrodynamics, the determination of the rate of propagation is made to depend on the conditions of the arbitrary mode of disturbance, whereas, as matter of fact, the propagation and rate of propagation are both independent of the particular form of disturbance. This contradiction of the theory by experimental facts sufficiently accounts for the reductio ad absurdum, which, consequently, signifies that the theoretical reasoning is at fault and requires to be rectified. To show how this is to be done is the purpose of the following investigation.

The problem to be solved is to account for the fact of a rectilinear transmission of effect from a disturbance marle at a certain point $A$ to any point $P$ in the surrounding fluid, without reference to any assigned mode of disturbing the fluid. Since there are no arbitrary initial conditions of the motion, it follows that the kind of motion must be indicated by some general analytical circumstance. It will here be supposed that the required analytical circumstance is that the differential function $u d x+v d y+w d z$ is an exact differential in all cases. On this supposition it may be assumed that there will be a rectilinear axis of motion between $\mathrm{A}$ and $\mathrm{P}$, the motion relative to the axis satisfying the condition of the integrability of $u d x+v d y+w d z$. Also in order that there may be motion persistently along the axis, it is necessary that the condensation pertaining to the motion should be symmetrically disposed about the axis, so that both the condensation and consequent transverse velocity will be functions of the distance $(r)$ from the axis. Consequently, supposing that the axis of $z$ coincides with the given axis of motion, we have to assume that, with reference to that axis,

$$
(d \cdot f \phi)=u d x+v d y+w d z
$$

$f$ being a function of $r$, and $\phi$ a function of $z$ and $f$. Accordingly,

$$
u=\phi \frac{d f}{d x}, \quad v=\phi \frac{d f}{d y}, \quad w=f \frac{d \phi}{d z}
$$


Since $f$ is a function of $r$-that is, of $\left(x^{2}+y^{2}\right)^{\frac{1}{2}}$-we have $\frac{d f}{d x}=\frac{x}{r}, \frac{d f}{d y}=\frac{y}{r}$; and hence, for any point on the axis, $u=0$ and $v=0$, as is required by the foregoing supposition. Also, because for every point on the axis $\frac{d f}{d x}=0$, and $\frac{d f}{d y}=0$, itfollows that $f$ has at each point a maximum or minimum value, which, in the absence of determining conditions, we may assume to be unity. Hence the velocity $w$ along the axis will be expressed by $\frac{d \phi}{d z}$.

From these results, taken in conjunction with the first and second general hydrodynamical equations, we might proceed to obtain actual values, expressed in series, of the quantities $f$ and $\phi$, from which the motions along and parallel to the axis, and in the directions transverse to the axis, might be inferreds. It is not necessary to introduce here these investigations, since I have already given them at length in arts. 20-28 of a communication, "on the Mathematical Theory of the Vibrations of an Elastic Fluid," inserted in the Philosophical Magazine for August 1862, and also in my work on the Principles of Mathematics and Physics, pp. 201-211. It is, at present, chiefly of importance to remark that, as I have been careful to point out, all this investigation is prior to the supposition of any arbitrary method of putting the fluid in motion, and consequently has exclusive reference to laws of the mutual action of the parts of the fluid irrespective of arbitrary conditions ; or, as I have said in the present communication, the motion is free, and spontaneous as to its laws.

Proceeding now to cases of arbitrary disturbance, it is first to be stated that, as the circumstances of spontaneous motion were determined prior to any discussion of arbitrarily imposed motion, the treatment of the latter must take into account the laws obtained relative to spontaneous motion. Accordingly the result of any arbitrary disturbance must be supposed to be a composition of spontaneous motions unlimited as to number and directions, and originating at the place of disturbance. The component motions may be designated as elementary motions, which, at the same time that they satisfy by their composition the given conditions of the disturbance, severally obey the laws of spontaneous motion obtained as above stated. They are capable of this application on account of their being derived from linear differential equations with constant coefficients. Since the position of the point $\mathrm{P}$ was taken ad libitum, what has been argued relatire to the resulting 
motion at $\mathrm{P}$ applies to every portion of the fluid surrounding A. Here, then, is the explanation of that diffusion of the effect of any disturbance which it was proposed to account for.

The rate of transmission of the effect of the disturbance has to be calculated from the laws of the elementary motions, without respect to any arbitrary mode of disturbance. This I have done by the solution of Proposition XIV. in pages 214224 of my treatise on the Principles of Mathematics and Physics, obtaining thereby the result that the theoretical value exceeds the observed value by 17.5 feet. The excess may with probability be ascribed to the circumstance that the air is not, as supposed in the theory, a simple fluid of perfect elasticity, but composite and loaded with vapour.

It would appear from the foregoing argument that the method of determining velocity of propagation in an elastic fluid from assumed conditions of the motion of the fluid, which was first adopted by Newton, and afterwards virtually followed by Lagrange and Poisson, is defective in principle, and that the exact method must be derived from the general equations of fluid-motion. Also it seems that the rapid alternate generations of heat and cold in a series of aerial vibrations does not, as has been generally supposed, sensibly affect the rate of propagation.

The results above obtained relative to the diffusion of sound by aerial vibrations equally pertain to the diffusion of light by ætherial vibrations, on the hypothesis, which I adopt, that the æether is a perfect fluid defined by the equation $p=a^{2} \rho$, $a^{2}$ being absolutely constant.

The motion of the air defined by the series for the function $\phi$, obtained in the manner already stated, is required for ascertaining the character of spontaneous aerial vibrations pertaining to the phenomena of sound, and for obtaining the laws of the harmonic series which is applied in the theory of music.

The motion of the æther defined by the spontaneous series for $f$, obtained as before stated, together with that for $\phi$, is necessary for ascertaining the character of a ray of light, the laws of transverse vibrations and of polarization, and the mode of derivation of a polarized ray from a ray of common light.

I think it right to mention here that the assertion that the integrability of $u d x+v d y+v d z$ indicates rectilinear motion, which I made at an early stage of my hydrodynamical researches, is adverted to by Professor Stokes in his Report on hydrodynamics contained in his recently published 'Mathematical and Physical Papers,' vol. i., and is with good reason objected to in page 161. I had not, at that time, arrived at the conception of 
the important distinction, indicated in the present communication, between spontaneous motion and motion produced under arbitrary conditions. The above assertion applies only to spontaneous motion. The recognition of this distinction suffices to meet completely Professor Stokes's objections.

At the end of a communication to the Philosophical Magazine for June 1880, I stated that I did not expect to have occasion to discuss any additional questions in Theoretical Physics. The importance and novelty of the present communication respecting spontaneous fluid-motion, the views respecting which have only very recently occurred to me, may, I think, be considered sufficient to justify my recurring once more to the subject of the Analytical Principles of Hydrodynamies.

Cambridge, February 21, 1881.

$\mathrm{XXXV.} \mathrm{On} \mathrm{the} \mathrm{new} \mathrm{Theory} \mathrm{of} \mathrm{Magnetic} \mathrm{Attractions,} \mathrm{and} \mathrm{the}$ Magnetic Rotation of Polarized Light. By H. A. RowLAND*.

TN a note published in the 'American Journal of Mathematics,' and also in the Philosophical Magazine for June 1880, I showed that the new action of magnetism on an electric current, recently discovered by Mr. Hall in my laboratory, was essentially of a rotational character, and I showed also that it was probably of the same nature as the rotation of the plane of polarization of light. I have since published a paper in the "American Journal of Mathematics"-" On the General Equations of Electro-magnetic Action, with Applications to a new Theory of Magnetic Attractions, and to the Theory of the Magnetic Rotation of the Plane of Polarization of Light "-in which the subject is treated in full, and Maxwell's formula for the magnetic rotation of the plane of polarization deduced from the newly discovered action of magnetism.

Mr. Hopkinson has recently drawn attention $\dagger$ to the fact that Maxwell has inserted a certain "Rotatory Coefficient" in his equations of resistance ('Electricity,' art. 303). Maxwell further states that the coefficient should be found, if anywhere, in magnets, or, of course, in any magnetic field, as it has now been found. But $I$ believe Maxwell nowhere connects this quantity with the rotation of the plane of polarization of light; and hence I think it may be well to give here an abstract of this portion of my paper.

In the 'Note' before referred to, I thought that it would be necessary to prove that the lines of electrostatic induction

* Communicated by the Author.

$†$ Phil. Mag. Dec. 1880, p. 430. 